\title{
Reflets
}

Revue d'intervention sociale et communautaire

\section{Retour sur un projet d'organisation communautaire axé sur la lutte à la pauvreté à Sudbury}

\section{Stéphanie Chevrette}

Volume 18, numéro 1, printemps 2012

URI : https://id.erudit.org/iderudit/1012338ar

DOI : https://doi.org/10.7202/1012338ar

Aller au sommaire du numéro

Éditeur(s)

Reflets, Revue d'intervention sociale et communautaire

ISSN

1203-4576 (imprimé)

1712-8498 (numérique)

Découvrir la revue

Citer cet article

Chevrette, S. (2012). Retour sur un projet d'organisation communautaire axé sur la lutte à la pauvreté à Sudbury. Reflets, 18(1), 182-189.

https://doi.org/10.7202/1012338ar d'utilisation que vous pouvez consulter en ligne.

https://apropos.erudit.org/fr/usagers/politique-dutilisation/ 


\section{Retour sur un projet d'organisation communautaire axé sur la lutte à la pauvreté à Sudbury}

\section{Stéphanie Chevrette}

Étudiante à la maîtrise en service social, Université Laurentienne

La problématique de la pauvreté affecte plusieurs individus et familles du Nord de l'Ontario. Dans cet article, nous nous proposons de faire un bref survol d'un projet d'organisation communautaire, Put Food in the Budget - De la nourriture dans le budget, relevant principalement d'un regroupement d'organismes de lutte à la pauvreté. Ce regroupement a lancé en 2010 le projet collectif le "Défi faites le calcul ", qui s'est déployé partout en Ontario, et plus spécialement dans la ville du Grand Sudbury.

Ce projet collectif est au cœur de nos études de maitrise en service social parce qu'il s'inscrit dans une perspective provinciale et communautaire de lutte à la pauvreté. L'objectif du «Défi faites le calcul » était de créer une sensibilisation sociale et de rassembler les communautés en vue de favoriser une délibération collective sur le sujet de l'insécurité alimentaire, et cela, dans le but ultime d'augmenter les prestations de sécurité du revenu pour celles et ceux qui peinent à se nourrir de façon décente à Sudbury comme dans la province.

\section{La pauvreté}

Qui est responsable de la pauvreté? Est-ce l'individu? Est-ce le gouvernement - ou l'État? Est-ce la communauté? Ces questions, 
qui nous interpellent depuis longtemps comme travailleuse sociale, peuvent être débattues de plusieurs façons. Mais selon Bourque, et collab. (2007, p. 159), " la pauvreté est avant tout un problème de société; elle découle d'une mauvaise répartition des ressources attribuables à la mondialisation des marchés et au désengagement de l'État ». Au fait, qu'en est-il de la pauvreté à Sudbury?

Près de 158000 personnes vivent dans la ville du Grand Sudbury et $14,9 \%$ d'entre elles se retrouvent en situation de pauvreté (Ville du Grand Sudbury, 2006). D'après les recherches effectuées par les bénévoles de Put Food in the Budget - De la nourriture dans le budget (2010), une personne bénéficiant de l'assistance sociale recevait un montant de $585 \$$ par mois en 2010. Une personne bénéficiant du programme ontarien de soutien pour les personnes handicapées recevait $1042 \$$ par mois. Le rapport sur le sans-abrisme du Réseau des sans-abri (2008) mentionne qu'un studio à Sudbury coûte en moyenne $485 \$$ par mois et un logement à une chambre coûte 651 \$. Enfin, Le panier à provisions nutritif, un rapport du Service de santé publique de Sudbury et du district Sudbury (2010), affirme qu'une personne doit dépenser 248 \$ par mois pour se nourrir adéquatement.

\section{Le «Défi faites le calcul»}

Comme les chiffres sur le coût de la vie montrent qu'une personne assistée sociale ne peut subvenir à ses besoins de base avec les allocations qu'elle reçoit, plusieurs organisations communautaires ont décidé de mettre sur pied un projet collectif s'inscrivant dans la lutte contre la pauvreté. Les groupes communautaires faisant partie de Put Food in the Budget - De la nourriture dans le budget ont signalé leur satisfaction quant aux changements apportés par la Loi de 2009 sur la réduction de la pauvreté en Ontario, mais ils voulaient prouver qu'il y avait toujours des problèmes et des besoins citoyens qui nécessitaient une intervention rapide du gouvernement. Ils ont alors fait la demande d'augmenter de $100 \$$ par adulte les montants octroyés par l'assistance sociale, et cela, dans le but de permettre aux personnes de se nourrir adéquatement. 
En lien avec leur demande d'augmentation des prestations d'assistance sociale, le collectif Put Food in the Budget - De la nourriture dans le budget a planifié une série d'activités communautaires, dont la première était un sondage visant à établir ce que doit dépenser chaque mois une personne seule pour répondre à ses besoins essentiels. Aussitôt terminé, ce sondage a été envoyé aux députés provinciaux afin d'obtenir leurs réactions. Bien que plusieurs réponses de députés indiquaient l'importance de hausser les prestations, la réponse de Madeleine Meilleur, alors ministre des Services sociaux et communautaires et député de la région d'Ottawa-Vanier, a étonné, même choqué, plusieurs personnes :

" [Mme Meilleur] comprend que les bénéficiaires d'assistance sociale ne reçoivent pas assez et qu'elle « aimerait bien ajouter un $100 \$$ par mois » pour leurs prestations, mais que « cela coûte cher à chaque fois que nous nous déplaçons sur les demandes de ce genre ", tout en reconnaissant le lien que la recherche démontre entre la santé et la pauvreté. Mme Meilleur dit que le public appuie les priorités du gouvernement en matière de santé, d'éducation et de l'économie et que l'aide sociale "n'est pas sur son radar ».» (Social Planning Network of Ontario - Réseau de planification sociale de l'Ontario, 2010, p. 6)

La déclaration publique de la ministre des Services sociaux et communautaires a mené le collectif Put Food in the Budget - De la nourriture dans le budget à planifier une deuxième activité, le "Défi faites le calcul ». Ainsi, pendant une semaine, on a invité des gens de tous les milieux sociaux à se nourrir uniquement de denrées provenant d'un panier d'une banque alimentaire. L'objectif de cette demande particulière était de simuler le plus justement possible une situation de pauvreté sans perturber les obligations quotidiennes des participants. L'activité a débuté à Toronto en avril 2010 avec dix participants ${ }^{1}$. Devant le succès de cette première expérience et afin de viser plus de gens, Put Food in the Budget - De la nourriture dans le budget a demandé aux autres 
communautés de l'Ontario de participer au projet. Ainsi, plus de dix communautés, dont celle du Grand Sudbury, ont participé au "Défi faites le calcul ». L'activité a connu un succès partout en Ontario. À Sudbury, 23 personnes y ont participé.

\section{Les résultats de la lutte}

Le comité organisateur du "Défi faites le calcul » de la région de Sudbury était composé de personnes qui se préoccupent de la problématique de la pauvreté ou assistées sociales. Du $1^{\text {er }}$ au 8 octobre 2010, 23 personnes de la région de Sudbury ne se sont alimentées qu'à la banque alimentaire. Les participants provenaient d'une variété de secteurs : santé, éducation, arts et culture, médias et secteur privé. Au cours de la semaine, les gens devaient respecter les consignes suivantes soit :

1. ne manger que ce qu'il y avait dans un panier venant de la banque alimentaire, soit l'équivalent de trois jours de nourriture - que les gens bénéficiant de l'assistance sociale doivent souvent étirer sur plus de dix jours;

2. participer à un souper communautaire;

3. emprunter les transports publics pour se déplacer;

4. partager leurs impressions et points de vue sur des blogues prévus à cette fin - www.dothemathchallenge.blogspot. com.

Le sujet le plus récurrent dans les discussions des participants sur les blogues aborde la question de la santé physique et mentale. Ainsi, les participants rapportent qu'ils se sentaient souvent faibles ou malades, car ils n'avaient pas accès à des aliments frais comme des fruits et légumes. Ces mêmes participants abordaient souvent les changements qu'ils observaient quant à leur humeur; plusieurs ont dit qu'ils avaient perdu beaucoup de motivation face aux activités quotidiennes. Ce constat du lien entre l'alimentation et la santé - physique et mentale - a permis à plusieurs participants de prendre conscience de la réalité de celles et ceux qui n'ont pour tout revenu qu'une prestation de la sécurité sociale et qui ont recours aux banques alimentaires. Par exemple, certains ont 
davantage compris pourquoi des jeunes vivant dans des conditions de pauvreté ont de la difficulté à se concentrer à l'école.

Rappelons qu'un des buts du "Défi faites le calcul » était de sensibiliser une population qui ne vit pas habituellement dans la pauvreté. Mais même si les participants qui ont accepté de relever le défi étaient déjà sensibilisés à la problématique de la pauvreté, nous avons pu constater par leurs commentaires sur le blogue que le but de l'exercice avait été atteint. Voici le témoignage d'une participante qui souligne sa prise de conscience :

"Le défi n'était pas seulement de créer une sensibilisation quant aux dons faits aux banques alimentaires ou du stigma que vivent les gens sur l'assistance sociale, mais plutôt qu'à la fin de la journée que nous sommes chacun des humaines et nous ne devrions pas offrir une aide parce qu'une personne est démunie, mais plutôt offrir une aide, car c'est une personne comme nous et elle aussi a le droit au respect et à la dignité. »

D'après les propos des participants au "Défi faites le calcul » recueillis sur les blogues, on note qu'en plus d'une prise de conscience chez plusieurs d'entre eux, il y a une volonté de participer au changement social. En effet, lors d'une session d'évaluation réunissant les participants et les membres du comité organisateur, on a pu constater une vision plus empathique face à la pauvreté. La plupart des participants ayant vécu l'expérience disaient que leur perception de celle-ci s'était véritablement transformée. L'activité d'évaluation a aussi permis de trouver des stratégies pour réduire la pauvreté. En voici quelques-unes :

- Augmentation des prestations d'assistance sociale afin qu'elles reflètent le coût réel de la vie;

- Plus de nourriture fraîche et "santé " aux banques alimentaires - fruits, légumes, pain de blé entier, etc.;

- Plus de jardins communautaires où les gens à faible revenu peuvent produire leur propre nourriture; 
- Partage de recettes dans les banques alimentaires afin que les gens puissent avoir de meilleures idées sur la façon de préparer certains plats.

Bien que le "Défi faites le calcul » se soit avéré un exercice intéressant ayant un impact sur plusieurs personnes partout dans la province, une critique peut toutefois s'adresser à la démarche communautaire en tant que telle : elle porte sur le manque d'un suivi politique significatif. En effet, la vie a trop facilement repris son cours, les participants retournant à leurs habitudes alimentaires et les gens qui bénéficient de l'assistance sociale demeurant encore et toujours aux prises avec les mêmes contraintes socioéconomiques et alimentaires. Personnellement, nous avons l'impression que le regroupement d'organismes pour la lutte à la pauvreté n'a pas su profiter de l'impact de l'exercice aux niveaux provincial et municipal.

De plus, on a porté une trop grande attention sur les banques alimentaires, leur financement, leurs besoins, alors que le but du "Défi faites le calcul " n'était pas d'éclairer les besoins des banques alimentaires, mais bien de faire comprendre qu'une augmentation décente des prestations d'assistance sociale pourrait faire en sorte que les citoyennes et citoyens qui vivent dans la pauvreté pourraient s'approvisionner comme tout le monde dans les marchés alimentaires et avoir accès à de la nourriture de qualité.

En revanche, et malgré ces critiques, on pouvait observer à Sudbury à la veille des dernières élections provinciales plusieurs affiches qui arboraient des slogans pour un "Ontario sans pauvreté ". Cela est-il un signe qu'il y a, à Sudbury, une plus grande sensibilisation citoyenne face à la pauvreté?

\section{Conclusion}

La lutte contre la pauvreté qui s'est actualisée par le "Défi faites le calcul " souligne l'importance du rôle de chacun dans l'élimination de la pauvreté. Dans le rapport gouvernemental, 
Rompre le cycle (2009 p. 2), la ministre de la Santé et des Soins de longue durée, Deb Matthews, indiquait que le gouvernement a évidemment un rôle à jouer. Elle lance le défi aux communautés d'y participer aussi.

Il s'agit d'un message important, car les individus ont un rôle à jouer dans l'élimination de la pauvreté, qui est un problème collectif qui nécessite une réponse collective. L'État et ceux qui le financent, les contribuables, les citoyennes et les citoyens, doivent s'assurer que les personnes et les familles dans le besoin puissent avoir accès à un niveau de vie décent et que leurs besoins essentiels soient satisfaits. Le "Défi faites le calcul » a permis de constater, dans le contexte de la mauvaise nutrition qui touche trop de gens à Sudbury et en Ontario, que l'inadéquation de la redistribution des richesses peut avoir un impact sur les activités de la vie quotidienne, sur la santé physique et mentale et sur le bien-être de nombreuses personnes vivant dans la pauvreté. L'activité a aussi donné cette précieuse occasion de réunir la communauté afin de trouver des solutions communes pour aider les plus vulnérables, pour faire la promotion d'une vraie expérience humaine l'égalité et le vivre ensemble. Bref, un petit pas dans les grandes démarches visant à lutter contre la pauvreté en Ontario.

\section{Note}

1 Les témoignages des participants peuvent être lus sur le site suivant : http://dothemath.thestop. org/dothemathchallenge_updates.php.

\section{Bibliographie}

BOURQUE, Denis, et collab. (2007). L'organisation communautaire, Québec, Presses de l'Université du Québec, 534 p.

CONSEIL NATIONAL DU BIEN-ÊTRE SOCIAL (2011). Le sens des sous pour résoudre la pauvreté, Ottawa.

DOUCET, Laval, et Louis FAVREAU (dirs.) (1994). Théories et pratiques en organisation communautaire, Sainte-Foy, Presses de l'Université du Québec, 468 p.

GOUVERNEMENT DE L'ONTARIO (2010). Rompre le cycle : stratégie pour la réduction de la Pauvreté, Toronto.

PUT FOOD INTHE BUDGET (2010). «Do the Math : Does It Add Up? », réf. du 7 février 2011, http://dothemath.thestop.org/doesitaddup.php 
RÉSEAU DES SANS-ABRI (2008). Rapport sur le sans-abrisme, Sudbury.

SOCIAL PLANNING NETWORK OF ONTARIO (2010). Do the Math MPP Visit Report, Toronto.

SERVICE DE SANTÉ PUBLIQUE DE SUDBURY ET DU DISTRICT (2010). Le panier à provisions nutritif, Sudbury.

VILLE DU GRAND SUDBURY (2006). Démographie, réf. 2011, http://www.greatersudbury.ca/ keyfacts/index.cfm?app $=$ KEYFACTSFR $\& \mathrm{ct}=414 \& \mathrm{lang}=\mathrm{fr}$ 